\title{
Analysis of Heart Rate Variability During a Mental Image Session of a Walk*
}

\author{
Jonathan Martinez*" \\ Laurent Mourot***
}

Recibido: marzo 13 del 2020 • Aceptado: mayo 04 del 2020

Abstract

The study of the autonomic nervous system (ANS) has become relevant due to its response during sports training. Exercise itself improves ANS status, but overtraining induces a state of chronic fatigue, unbalancing ANS activity. Therefore, it is necessary to find other methods to improve the sporting performance. The mental image (MI) has been shown as an effective method to improve the performance of certain motor conditions in athletes, exhibiting the activation of the ANS. MI sessions could be used as a training method; thus, the objective of this research was to identify if there is a significative activation of the ANs during and after MI sessions. The heart rate of 15 subjects was monitored during a physical session and during an MI session of a walk in two different speeds. Data was analyzed through ANOva and it did not show significative changes in the heart rate variability during or after the MI. It's

Research article, self-financed, associated to the unit EA3920 Prognostic Markers and Regulatory Factors of Cardiovascular Diseases and Exercise Performance, in the Exercise Performance Health, Innovation Platform, Besançon, France.

How to cite: Martinez, J. and Mourot, L. (2020). Analysis of heart rate variability during a mental image session of a walk during walk mental imagery. Revista de Investigación Cuerpo, Cultura y Movimiento, 10(2), 97-118. DoI: https://doi.org/10.15332/2422474x/6229

** Master in training and optimisation of sport performance, Research Assistant, Exercise Performance Health, Innovation Platform, Besançon, France. jonathan94.mar@gmail.com. ORCID: 0000-0003-2592-1888

*** PhD, Research Unit EA3920 Prognostic Markers and Regulatory Factors of Cardiovascular Diseases and Exercise Performance, Manager Exercise Performance Health, Innovation Platform, Besançon, France. laurent.mourot@univ-fcomte.fr. ORCID: 0000-0001-9486-3090 
possible that the result of this research was influenced by the mental effort, the lack of training in MI, and the time used to obtain the data to analyze the heart rate variability.

Keywords: autonomic nervous system, autonomous activation, high frequency index, parasympathetic activation. 


\section{Análisis de la variabilidad de la frecuencia cardiaca durante la imagen m laaminata}

\section{Resumen}

El estudio del sistema nervioso autónomo (SNA) ha cobrado relevancia debido a su respuesta durante el entrenamiento deportivo. El ejercicio mejora el estado del SNA, pero el sobreentrenamiento puede inducir un estado de fatiga crónica, desequilibrando su actividad. Por lo tanto, es necesario encontrar otros métodos para mejorar el rendimiento deportivo. La imagen mental (IM) es un método eficaz para mejorar el rendimiento de ciertas condiciones motoras en los atletas y ha demostrado la activación del SNA. Las sesiones con IM podrían ser utilizadas como método de entrenamiento, por lo que el objetivo de esta investigación fue identificar si hay activaciones significativas del SNA durante y después de las sesiones de IM. Se monitorizó la frecuencia cardiaca (HR) de 15 sujetos durante una sesión de caminata tanto física como por IM en dos velocidades. Los datos fueron analizados por medio de ANOvA, que no mostró cambios significativos en la variabilidad de la frecuencia cardiaca durante o después de la sesión de im. En conclusión, es posible que el resultado de esta investigación esté influenciado por el esfuerzo mental, la falta de entrenamiento en IM y el tiempo para obtener los datos de la variabilidad de HR.

Palabras clave: sistema nervioso autónomo, activación autónoma, índice de alta frecuencia, activación parasimpática. 


\section{Introduction}

The study of the autonomic nervous system (ANS) has become relevant due to the response of various systems to external stimuli or workloads during physical activity or sports training (Borrensen \& Lambert, 2008).

Regular physical activity allows the improvement of the general physical condition. In the cardiovascular system, specifically, it allows an increase in plasma volume, the contractibility of the heart, and the volume of blood flow. It also allows an elevation of catecholamines, a decrease in systolic and diastolic blood pressure, and a decrease in the metabolic load of the heart during rest and physical activity. Finally, it contributes to the health of the ANs by decreasing the heart rate (HR) and increasing the heart rate recovery (HRR). (Aubert, Seps \& Beckers, 2003; Borrensen \& Lambert, 2008).

During physical activity there is an increase in the HR due to sympathetic activity followed by a state of recovery that creates equilibrium between sympathetic and parasympathetic activity in response to stimulation. A late recovery of the HR may mean that the function of the ANs is compromised and is related to a high mortality rate. In fact, an accelerated recovery of the HR demonstrates an optimal function of the autonomous response and a good cardiovascular condition (Borrensen \& Lambert, 2008). Correspondingly, high HR variability is associated with high oxygen consumption and with good ANs status, while low values are associated with cardiac events and risk of sudden death (Achten \& Jeukendrup, 2003; Lauer, 2009).

All the above benefits to the cardiovascular system are applicable to high-performance athletes. These athletes experience sinus bradycardia, increased capacity, and thickness of the left ventricular diastolic cavity in men and smaller left ventricular mass in women. This is defined as the athlete's heart. Additionally, athletes experience an increase in heart volume and a contraction resulting in the optimization of blood flow to the brain, as well as low heart rate due to long periods of diastole and submaximal oxygen uptake caused by a shift from sympathetic to parasympathetic activations of the ANs, although this last one is still under discussion (Aubert, Seps \& Beckers, 2003, pp. 900-901; Dong, 2016, p. 1532).

$\mathrm{HR}$ is the speed of the heartbeat per unit of time, calculated to assess the heart rate variability (HRV). It is obtained by recording the electrical signal of the heart. These recordings are then analyzed to extract the R-R wave 
intervals, which allow to identify the oscillations and the influence of the sympathetic and parasympathetic system (Dong, 2016, p. 1531; Pinto, Russo, Lemos, Domingues \& Aureliano, 2017, p. 190).

Recently, HRV was used during training as a marker to reflect the modulation of nerve pathways and to monitor the adaptation or overtraining of athletes; as a result, HRV allowed the configuration of adequate training loads and recovery times (Dong, 2016, p. 1532; Achten \& Jeukendrup, 2003; Aubert, Seps \& Beckers, 2003).

During training periods there is an increase in sympathetic signals that allow better performance; then, during recovery phases, there are physiological adaptations (Dong, 2016, p. 1532), such as a gradual improvement in parasympathetic activation that reflects an improvement in physical condition, allowing a shorter recovery time and better tolerance to workloads (Schmitt, Regnard, \& Millet, 2015, p. 2).

A high parasympathetic / low sympathetic activity reveals a good health status of the ANS and is thus of primary importance for athletes. Exercise itself improves ANS status. One potential drawback with athletes is that they can perform too much activity that could ultimately trigger a state of fatigue, unbalancing ANS activity. Therefore, it is necessary to find other classic training methods to improve ANS status.

The mental image (MI) has been shown as an effective method to improve the performance of certain motor and psychological conditions in athletes. Therefore, it has been used in domains such as motor learning, sports performance, motivation, self-confidence, anxiety management, strategies and problem solving, and injury rehabilitation (Guillot \& Collet, 2008).

Several authors indicate that during MI sessions the ANs indicators such as heart rate (HR), blood pressure (BP), electro skin resistance (SR) and respiratory rate (RF) are activated (Oishi, Kasai, \& Maeshima, 2000, p. 256; Kazuo \& Takashi, 2004; Guillot \& Collet, 2005; Demougeot, Normand, Denise, \& Papaxanthis, 2009; Collet, Di Rienzo, Hoyek \& Guillot, 2013; Pinto, Russo, Lemos, Domingues \& Aureliano, 2017), partly because autonomous activations are present during real activity given the neuronal connection of the somatic nervous system in charge of movement and the aNs.

It was possible to relate activations of ANs indicators caused by the regional cerebral blood flow; studies show that during the MI of a motor activity there is an activation of the cerebellum, the basal ganglia, and the cerebral 
motor area (Kazuo, Kasai \& Maeshima, 2000). Thus, more recent neural studies support that there is an important connection between brain circuits involving imagination and nervous system responses (Pinto, Russo, Lemos, Domingues \& Aureliano, 2017).

Several authors reported that during the MI sessions of various body movements such as hip flexion, vertical jump, shoulder flexion, standing hamstring stretch, trunk elevation and leg elevation at $90^{\circ}$ from a supine position, 500 meter sprint in skating or sports training lifting two machine loads, among others, there are relevant activations of HR, PA, and RF during these MI sessions compared to the real sessions. These sessions are composed of various repetitions and have an approximate duration ranging from 5 seconds to 45 seconds (Decety, Jeannerod, Durozard, \& Baverel, 1993; Kazuo \& Takashi, 2004; Pinto, Russo, Lemos, Domingues \& Aureliano, 2017).

It has been suggested that these activations in the ANS, by means of MI, are due to the function of anticipation or motor programming from the higher brain centers (central command). However, it is not yet clear how these factors intervene during MI (Oishi, Kasai, \& Maeshima, 2000; Kazuo \& Takashi, 2004).

A precise definition of mental image was written by Morris et al. (2005). Mental images in the context of sport can be considered as the creation of an experience at the mental level generated from pre-existing information in memory, involving quasi-sensory, quasi-perceptual, and quasi-affective characteristics, which are under control of the brain imager, and which can occur in the absence of real preceding stimulation.

This mental representation is associated with real experience (Guillot \& Collet, 2008) without producing muscular activity, allowing the person to feel the sensations that exist during muscular contraction due to cerebral plasticity, and by means of a cortical activation in the brain (Collet, Di Rienzo, Hoyek \& Guillot, 2013). This cerebral plasticity will alert the brain to carry out restructuring by means of the cortical way to increase physical capacities and using the subcortical way to increase the performance of the movements (Collet, Di Rienzo, Hoyek \& Guillot, 2013).

Consequently, due to the general connection of the nervous system to interpret the stimulation and generation of responses, it is necessary to plan and program the motor execution. All this happens according to an internal model of movement; in the case of MI, memory will allow the generation of 
the motor images from the same internal model (Collet, Di Rienzo, Hoyek \& Guillot, 2013).

The execution of the internal model during MI will generate the activation of the ANS indicators. Specifically, it can be seen an increase in HR (this is one of the most sensitive indicators to external stimulation). This implies an activation of the autonomous control and an elevation of the sympathetic tone during $\mathrm{MI}$ and subsequently an elevation of the parasympathetic tone during recovery. This activation can be measured continuously by noninvasive instruments, although the physiological response will not be of equal power (Guillot \& Collet, 2005).

Since MI sessions could improve the technique from a mechanical perspective of the planning and programming of complex movements if it is possible to demonstrate that there are autonomous responses by means of HRV during these sessions, it could be possible to have cardiovascular implications without producing physical fatigue in the athlete. In addition, MI sessions could be used as a training method and in the preparatory moments before the competition. Therefore, the objective of this research was to identify whether during and after MI sessions there are significant changes in autonomous activations of HRV.

\section{Method}

\section{Subjects}

This study is an experimental, descriptive quantitative study of a nonprobabilistic sample. Fifteen healthy and moderately active male volunteers were evaluated (age, 30, $13 \pm 10,77$ years; height, $1.77 \pm 0.06 \mathrm{~m}$; weight, $75.2 \pm 10.08 \mathrm{~kg}$ ). An explanation of the interventions to which they would be subjected was then made, with their consent to participate in this study. The participants followed certain recommendations before attending the interventions. Participants were unable to engage in strenuous physical activity 12 hours before the intervention, they had to comply with at least 7 hours of restorative sleep, and avoid the consumption of liquor, caffeine, and tobacco. 


\section{Experimental Design}

The subjects carried out two sessions of 1 hour and 30 minutes on two consecutive days, from midday onwards and at the same hour. The sessions were called real session (REE) and mental image session (MI), each divided into two 30-minute moments with a 30-minute rest.

Real session (REE)

The participant is welcomed and, before beginning with the installation of instruments, he is again warned of his right to withdraw the study at any time without incurring in any liability or injury because of it. Likewise, he is reminded that the data obtained are noninvasive and of the protocol to be followed.

The protocol starts with the setting of the HR monitor to record the HR. Then the person is asked to sit in a chair. The exercise starts with a timed rest of $3 \mathrm{~min}$, this to stabilize the HR and reduce the stress of the subject before starting to record the data.

HR recording starts with the subject seated watching an animal's video for 10 minutes. After that time, the subject receives an acoustic signal that tells him to stand up and walk at least 40 meters back and forth for 5 minutes at a normal walking speed. Afterwards, a new sound signal tells the subject to return and seat on the chair, resume the video, and remain in a resting position for 15 minutes. This is the end of the first part of the protocol.

After half an hour of break, the same protocol is repeated increasing the speed of the walk. In this second part of the session, the participant is asked to increase the walking speed, that is to say that the participant must make an increasing speed of the exercise in comparison wtih the first part of the protocol (there is not a determined speed), the participant cannot run, but the walking speed must be faster than the first session, during the 5 minutes of the evaluation.

\section{Mental Image sessions (MI)}

It is performed at the same time and length and in the same order as the previous REE session. The same starting conditions are respected (initial sitting position, setting of the HR monitor). During the practical part, the subject is asked to remain seated. One minute before starting this part, the video is 
stopped and the participant is asked to close his eyes. When the participant hears the sound signal, he must start imagining that he gets up from the chair and begins to walk at a normal speed making the same route of the previous day around two cones at a comfortable speed during 5 minutes. One more time, the beep tells the participant when to return to a seated position. Finally, the subject opens his eyes and resumes the video.

Table 1. Chronology of the REE and MI intervention

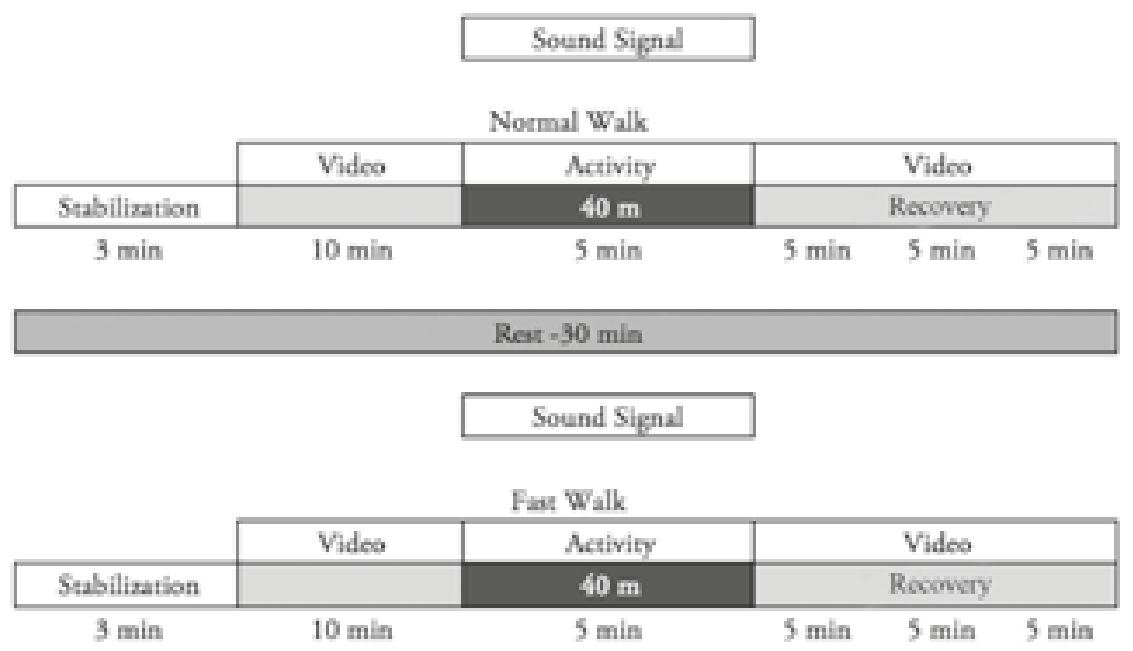

Source: Author's own creation.

Data Acquisition and Statistical Analysis

To perform an analysis of ANS activity on HR, it is necessary to analyze the time variation between R-R intervals of HR, called analysis of HRV (Aubert, Seps \& Beckers, 2003, p. 892; Scott, Graham, \& Davis, 2017, p. 2).

The HRV is divided into the time and frequency domain, several indices compose each domain and its interpretation allows to identify the autonomous control. The most important indices for this research are the SDNN (standard deviation of the R-R interval), which determines the variance of the 
R-R intervals; the r-MSSD (square root of the difference of the squares of the successive R-R interval), which quantifies the cardiac vagal control; the low frequency (LF) which, for some authors, can be interpreted as sympathetic activity and for others it can be interpreted as an interaction between sympathetic and parasympathetic activations; the high frequency $(\mathrm{HF})$ index, which is a reflection of parasympathetic activity; and finally, the ratio LF/HF that reflects a sympathetic-vagal relationship, although the r-MSSD and LF indices are still widely discussed (Achten \& Jeukendrup, 2003).

For data acquisition, the Polar S810i heart monitor was used along with the T61 heart belt, which is validated to perform an appropriate measurement of the R interval time fluctuation (Gamelin, Baquet, Berthoin, \& Bosquet, 2006).

The data was exported to Kuvios HRv Standard Ver. 3.2 for Windows. This program was used to visualize the curve of the data, to make a correction of the signal artifacts, and to obtain the values of each segment of the recorded data (Gamelin, Berthoin, \& Bosquet, 2005; Scott, Graham, \& Davis, 2017; Tarvainen, Lipponen, Niskanen, \& Ranta-aho, 2019).

Each 300-second segment of the recorded data corresponds to the rest phase (Rps), the physical exercise (Ex), and the three moments of recovery $(\mathrm{R} 1,2,3)$. The total data recorded was 1500 seconds per session. For its analysis in the time domain, values of the r-MSSD index and, in the frequency domain, the LF spectrum (LF), the HF spectrum (HF), and the LF/HF ratio were considered as the most significative.

Finally, all the results were exported to Excel to perform a standard statistical analysis and to calculate the mean and standard deviation of the values obtained. Then a comparison was made between the REE and MI conditions during the normal and fast walk. To analyze the variance, the statistical test ANOVA was used to compare MI and REE conditions during the segments of the protocol. The significance value considered for this study was $\mathrm{p}<0.05$.

\section{Results and discussion}

The mean values and standard deviation of HRV indices represent the ANS activations during normal and fast walking. The figures represent the results obtained for each index, as well as the REE or MI conditions. Figures 1 to 4 represent the normal walk and figures 5 to 8 represent the fast walk. 
Figure 1. HR - During Normal Walking

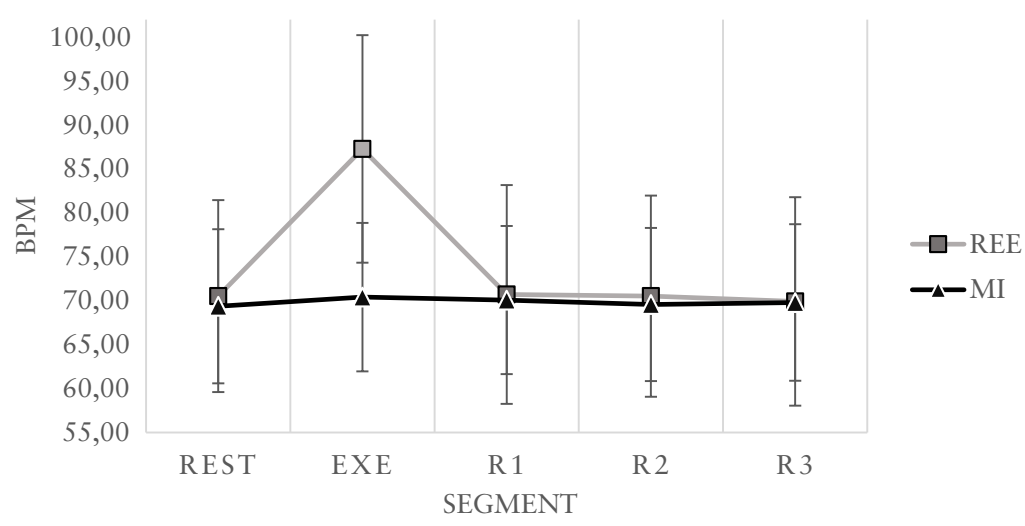

Source: Author's own creation.

Figure 2. r-MSSD - During Normal Walking

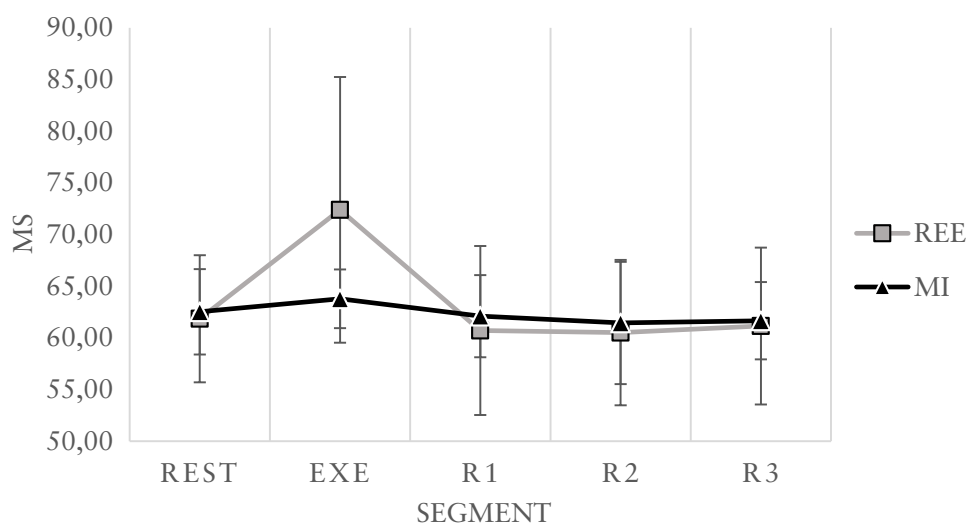

Source: Author's own creation. 
Figure 3 HF - During Normal Walking

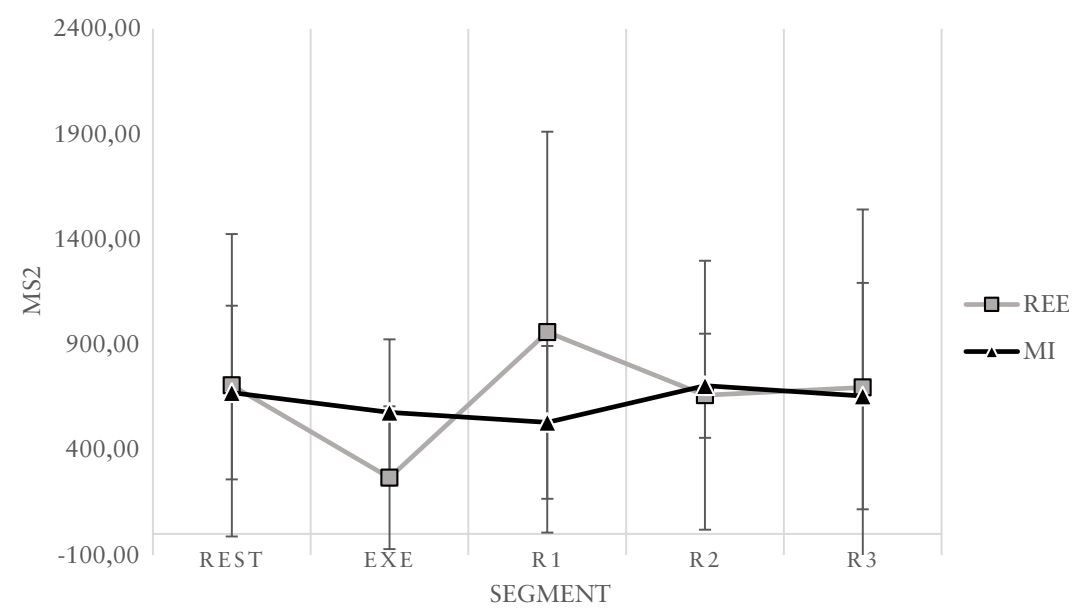

Source: Author's own creation.

Figure 4. Ratio LF:HF - During Normal Walking

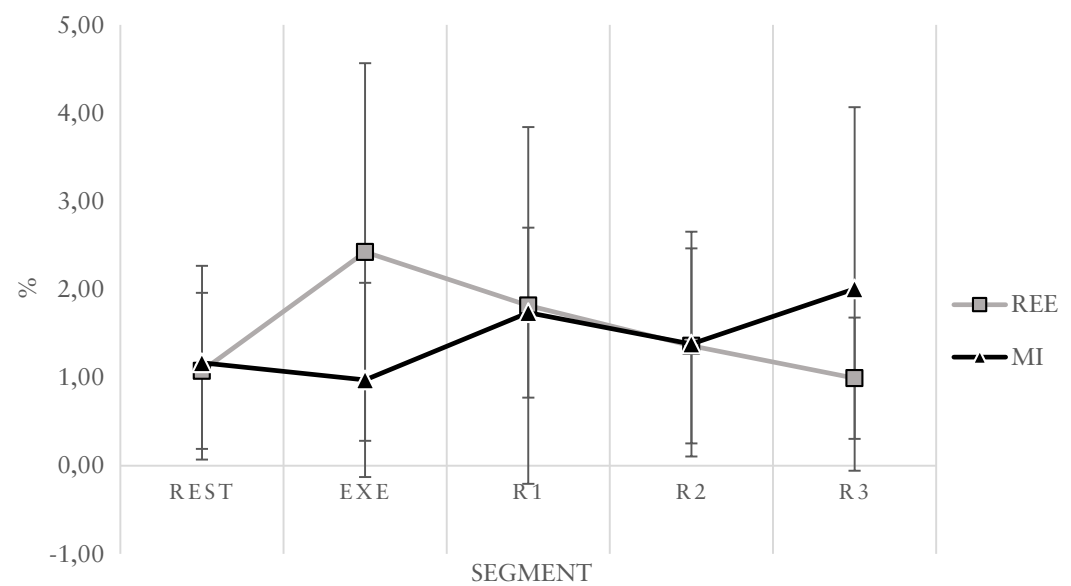

Source: Author's own creation. 
Figure 5. HR - During Fast Walking

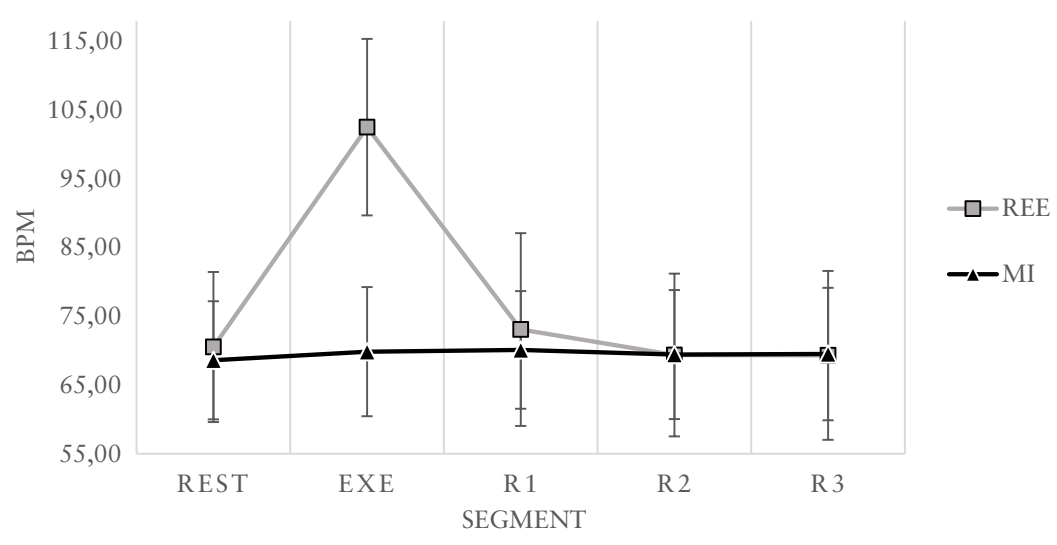

Source: Author's own creation.

Figure $6 r$-MSSD - During Fast Walking

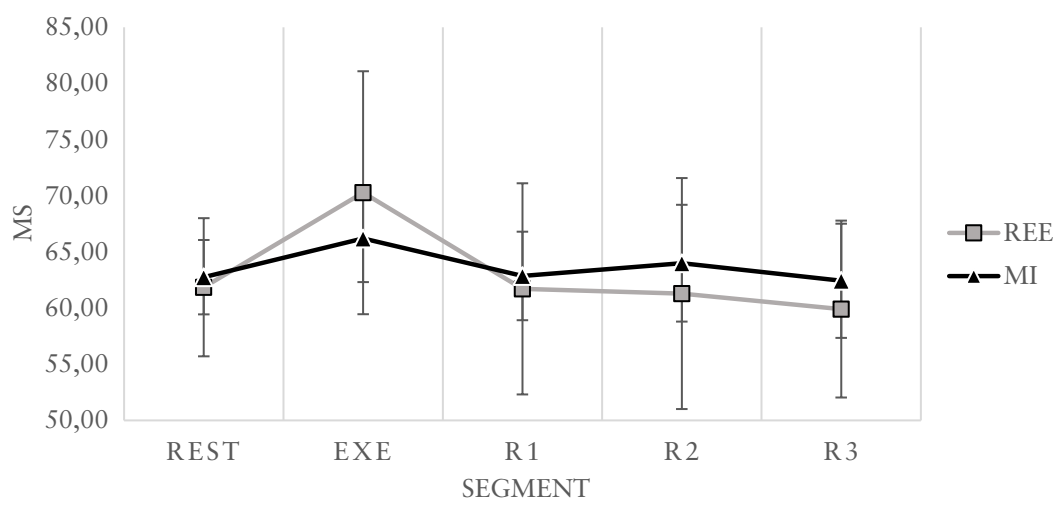

Source: Author's own creation. 
Figure 7. HF - During Fast Walking

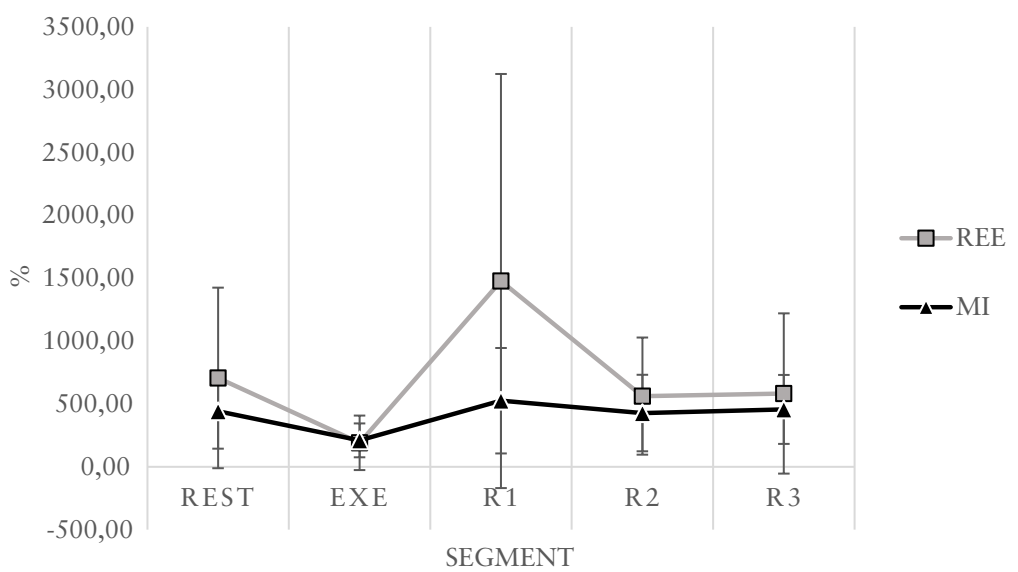

Source: Author's own creation.

Figure 8. Ratio LF:HF - During Fast Walking

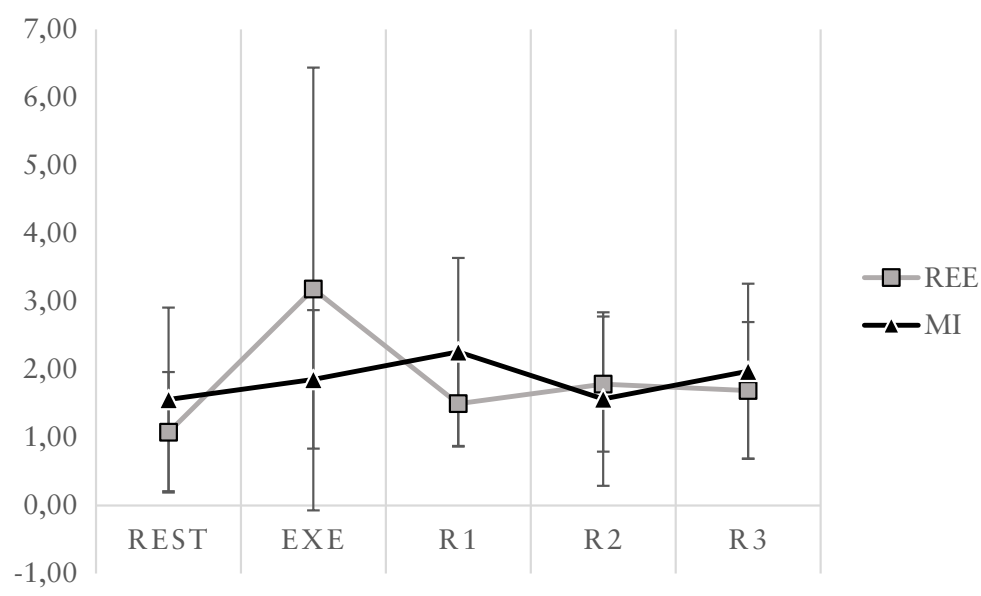

Source: Author's own creation. 
In neither of the two situations of gait speed major changes were noticeable in either HR, HF or LF:HF ratios. No significant decrease in parasympathetic activation during exercise segments was demonstrated (Figures 3 and 7), nor an increase in parasympathetic signal during the first recovery processes (Figures 3 and 7).

The ANOva analysis was performed to identify if there were significant variations in autonomic activations. The results of the analysis showed the expected significant variations between normal and fast walking. The behavior between protocol phases in the real condition was as expected. However, it did not show a significant variation between protocol phases during the imaginative condition.

The average results of the HRV indices do not show a significant increase in sympathetic values during the MI condition and the exercise phase (EXE) (Figures 2 and 6). Similarly, no significant increases in parasympathetic activation are found in the MI condition during the first recovery phase (R1) (Figures 3 and 7). All these results are proved by the ANOvA analysis.

This study seeks to identify whether, during the MI sessions of a habitual movement, activations of the sympathetic and parasympathetic branches of the ANS are achieved in relation to their activation during the real sessions. Several authors support the hypothesis that MI can generate an increase in HR and consequently an increase or decrease in HRV indices according to the phase during the protocol (Pinto, Russo, Lemos, Domingues \& Aureliano, 2017, p. 193).

Initially, it is said that there is a balance between the branches of ANs; during the stages of exercise the parasympathetic indices have a withdrawal behavior to give way to the sympathetic activation. After exercise, there is a blockage of the sympathetic signals, and the parasympathetic indices increase during the moments of rest to provide homeostasis (Borrensen \& Lambert, 2008; Schmitt, Regnard, \& Millet, 2015).

Therefore, it was hypothesized that if during MI sessions it was possible to increase the HR, this would be reflected in the HRV indices. However, the balance of parasympathetic values during the MI should resemble the behavior of the indices during the real situation.

However, the results of this research show an increase in HR during actual walking conditions, a decrease during the first resting phase, and a stabilization during the following two phases (Figures 1 and 5), this compared to 
the MI condition, where there weren't significant changes between the initial resting phase and the exercise phase (Figures 1 and 5). The values remain almost constant throughout the protocol.

On the other hand, the HRV showed that during the exercise phases HF has a withdrawal and later an increase according to the walking speed (Figures 2 and 6), this was compared with the r-MSSD and ratio LH:HF indices that showed increments during the exercise phases and a decrement during the recovery phases (Figures 7 and 8), confirming, thus, the authors' perspective. However, the indices during the MI condition remained almost constant during the exercise and recovery phases.

Comparing the real conditions with the imaginary ones, the HR and the HRV indices remain very stable and without significant changes between the phases of the protocol and regardless of the type of stimulus. The speed of the walk does not match with the results of previous studies, since two types of walking were performed with the aim of showing low and high activations based on the stimulus effort created, but without considering the intensity of the exercise, the values remain constant.

By means of experimentation processes, several authors have established three key factors to allow the use of MI: vivacity, accuracy, and control of the motor task (Collet, Di Rienzo, Hoyek \& Guillot, 2013). For this reason, during the real experiences a specific task was assigned to the subject; it was a habitual activity that could be controlled. Additionally, the participant was asked to be very attentive to all the sensations, sensitive and visual, during the walk. This because the MI will depend on the specific characteristics of the movement evoked from the memory to the generator center of images from a real situation.

On the other side, the magnitude of the activation of the autonomous effectors is proportional to the degree of mental effort during MI (Kazuo, Kasai, \& Maeshima, 2000). For this reason, it must be said that all the factors to perform a good MI were considered. However, the suggested stimulus or motor activity (walking) was probably not intense enough to provoke significant autonomous activations.

The above is related to the study of Kazuo, Kasai, \& Maeshima (2000) that establishes a similar protocol, comparing MI with a Mental Arithmetic (MA) test. This study was able to identify that the heart rate increased during the MI, and after the start of the task (sprint skate race of $500 \mathrm{~m}$ competition 
level) it continued to increase, achieving an increment of $24 \%$ to $77 \%$ of HR (from 13.9 to 53.9 beats/minute). The magnitude of the HR increase was significantly higher in $\mathrm{MI}$ than in $\mathrm{MA}(44.3 \% 18.8 \%$ in $\mathrm{MI}, 10.3 \% 4.3 \%$ in MA; $t$ 4.99, P 0.001). However, the test subjects were elite skating athletes, who have an MI training program incorporated into the macrocycles. All the athletes have had MI training from 5 to 60 months prior to the realization of the study. The study MI sessions were performed 4 times on different days, with a duration of 35 to 38 seconds. Only the MI values that would have had the best score were considered according to an evaluation that determines the vivacity and type of MI.

Comparing the previous study and the present study, it can be identified that the intensity and the activity that produce the stimulation can generate enough stress to achieve activations in the ANs, in contrast to other real exercises in activities such as load lifting, basic exercises, or more habitual movements (Decety, Jeannerod, Durozard, \& Baverel, 1993; Demougeot, Normand, Denise, \& Papaxanthis, 2009).

During the present study results show that after the MI exercise was started, the HR was not significantly increased. This may be caused in part due to the quality of the stimulus. Its stress must be able to stimulate the autonomic pathways and generate an increase in the subject' ANs markers already mentioned. The main stress condition is related to emotions, intensity of the external stimulus, and novelty; the ANs deals with the regulation of HR according to the quality of the stimulus, a moderating function is exercised by means of the valgus nerve and activity in the ambiguous nucleus of the central nervous system, where the processes of attention and emotional sensation are correlated. Any stimulation must be sufficiently intense to evoke a vegetative response (Collet, Di Rienzo, Hoyek \& Guillot, 2013).

Therefore, a MI session that seeks to obtain activations in the autonomous control of HR must generate considerable stress based on the individual's capacity for imagination and the subject's experience of performing the motor action. MI only relies on perceptions and emotions evoked by memory, supported by the connection between mental functions and the activation of the ANS.

The main benefit of $\mathrm{MI}$ is access to pre-existing information through the motor network, regardless of movement and as a result there are activations in the ANs (Gregg, Hall, \& Andrew Butler, 2010). The main problem of MI 
is the objective of the activity to be performed to generate an autonomous activation. Therefore, a complex and more strenuous motor task during MI can lead to a greater autonomous response (Oishi, Kasai, \& Maeshima, 2000).

It was also found relevant in the study by Kazuo, Kasai, \& Maeshima (2000) that the athlete's preparation in the use of $\mathrm{MI}$ is an important consideration, since more than $150 \mathrm{MI}$ training sessions are necessary during training to achieve clear images of a specific movement (Kazuo \& Takashi, 2004). 10-minute sessions are recommended to work on a skill and 20 minutes to manage anxiety (Guillot \& Collet, 2008). Other studies carried out with a similar methodology do not specify whether the athletes had any type of training or preparation in MI.

Also a study conducted by Fortes, et al. (2018) with the objective of demonstrating that $\mathrm{MI}$ training has repercussions in the effectiveness of the passes decision in volleyball players, demonstrated that in addition of the pass effectiveness improvement there is an increase of the HR during the MI $(F(2$, $32)=.94, p=.55)$. Therefore, it is established that even the training of athletes in MI must be paramount to find meaningful answers.

One of the main limitations of this study is the duration of MI: while the mental practice of motor tasks during MI allows the development of an ability to improve performance, the duration of motor task during MI is very similar to the real situation (Guillot \& Collet, 2010), as evidenced in Kazuo's study, Kasai, \& Maeshima (2000), where MI sessions have a duration of between 35 and 38 seconds that are compared to an athlete's average time to travel the required distance. During this time, due to the requested motor task and the athlete's MI training, the HR elevation is experienced.

The duration of the MI during this experimentation is too long and people tend to lose focus after a short time. Also, to be able to perform an effective HRV analysis, HR records of a minimum of 5 minutes are necessary. Considering this factor, the time is too low (Borrensen $\&$ Lambert, 2008; Tarvainen, Lipponen, Niskanen, \& Ranta-aho, 2019), therefore, the continuous imaging modality may not be the right one for the subjects; it is possible that intermittent sessions can get activations during the IM.

Other limitations by the side of the subjects are the capacity of imagination and the type of images that are possible during the MI. The capacity of imagination refers to the capacity of each person to develop mental images (Guillot \& Collet, 2008). Although each person has an individual capacity to 
imagine, some people will establish mental images clearer than others, which in turn translate into activations of the ANs. This capacity is also linked to the type of mental images, these are classified into two: kinesthetics images, which are experienced as if the person performed the activity in real time, based on kinesthetics information about movement, and visual images, those are experienced as if the person were a spectator of his or her movement (Pinto, Russo, Lemos, Domingues \& Aureliano, 2017, pp. 189-190).

Imagination capacity and type of mental image are aspects of MI training, which as explained above require planning during training seasons. Both aspects are evaluable by means of subjective scales.

Finally, since changes in HRV require interventions of at least three days a week, with a duration of 30 minutes, and activities with intensities higher than $60 \%$ of HRmax (Aubert, Seps \& Beckers, 2003) plus analyzing it from a perspective of sports training, we will propose that the motor tasks used for the MI have intensities higher than $60 \%$ of HRmax. These could show better autonomous activations during MI, in relation with searching the enough stimulation, time, stress, and mental effort of activities in sports training.

\section{Conclusion}

It was not possible to evidence significant autonomous activations during or after MI comparable to physical exercise. It is necessary to adapt the stimulus and the training of the subjects to achieve activations during the MI and during the moment of recovery. It is possible that in subjects trained in MI the expected activations will be achieved. However, although HRV is an effective method to identify the subtlest activations of the ANS, time is a limiting factor to use MI. 


\section{References}

Achten, J. \& Jeukendrup, A. (2003). Heart Rate Monitoring Applications and Limitations. Sports Med, 33(7), 517-538. DoI: https://doi.org/10. 2165/00007256-200333070-00004

Aubert, A., Seps, B. \& Beckers, F. (2003). Heart Rate Varibility in Athletes. Sport Med, 33(12), 889-919. DoI: https://doi.org/10.2165/00007256-200333120-00003

Borrensen, J. \& Lambert, M. (2008). Autonomic Control of Heart Rate during and after Exercise. Sports Med, 38(8), 633-645. DoI: https://doi. org/10.2165/00007256-200838080-00002

Collet, C., Di Rienzo, F., Hoyek, N. \& Guillot, A. (2013). Corrélats neurophysiologiques de l'imagerie motrice. Movement \& Sport Sciences, 82, 7-19. DOI: $10.1051 / \mathrm{sm} / 2010068$

Decety, J., Jeannerod, M., Durozard, D. \& Baverel, G. (1993). Central Activation of Autonomic Effectors during Mental Simulation of Motor Actions in Man. Journal of Physiology, 461(1), 549-563. DoI: https://doi.org/10.1113/jphysiol.1993.sp019528

Demougeot, L., Normand, H., Denise, P., \& Papaxanthis, C. (2009). Discrete and Effortful Imagined Movement Do Not Specifically the Autonomic Nervous System. PLoS ONE, 4(8), e6769. DOI: 10.1371/journal.pone.0006769

Di Rienzo , F., Blache, Y., Kanthack, T., Monteil , K., Collet, K., \& Guillot, A. (2015). Short-term effects of integrated motor imagery practice on muscle activation and force performance. Neurosciences, 305, 146-156. DoI: https://doi. org/10.1016/j.neuroscience.2015.07.080

Dong, J. (2016). The role of heart rate variability in sport physiology. Experimental and Therapeutic Medicine, 11, 1531-1536. DoI: https://doi.org/10.3892/ etm.2016.3104

Fortes, L. S., Freitas-Júnior, C. G., Paes, P. P., Vieira, L. F., Nascimento-Júnior, J. R. A., Lima-Júnior, D. R. A. A., \& Ferreira, M. E. C. (2018). Effect of an eightweek imagery training programme on passing decision-making of young volleyball players. International Journal of Sport and Exercise Psychology, 1-9. DOI: 10.1080/1612197x.2018.1462229

Gamelin, F., Baquet, G., Berthoin, S., \& Bosquet, L. (2006, August 08). Validity of the Polar S810 to Measure R-R Intervals in Children. Training \& Testing, 29(2), 134-138. DOI: https://doi.org/10.1055/s-2007-964995 
Gamelin, F., Berthoin, S., \& Bosquet, L. (2005). Validity of the Polar S810 Heart Rate Monitor to Measure R-R Intervals at Rest. Medicine \& science in sports \& exercise, 38(5), 887- 893. DOI: 10.1249/01.mss.0000218135.79476.9c

Gregg, M., Hall, C. \& Andrew Butler. (2010). The MIQ-Rs: a suitable option for examining movement imagery ability. ECAM, 7(2), 249-257. DoI: 10.1093/ ecam/nem 170

Guillot, A. \& Collet, C. (2008). Construction of the Motor Imagery Integrative Model in Sport: A review and theoretical investigation of motor imagery use. International Review of Sport and Exercise Psychology, 1(1), 31-44. DoI: 10.1080/17509840701823139

Guillot, A. \& Collet, C. (2005). Contributions from neurophysiological and psychological methods to the study of motor imagery. Brain Research Reviews, 50, 387-397. DOI: https://doi.org/10.1016/j.brainresrev.2005.09.004

Guillot, A. \& Collet, C. (2010). Duration of Mentally Simulated Movement: A Review. Journal Motor \& Behavior, 37(1), 10-20. DoI: 10.3200/jmbr.37.1.10-20

Kazuo, O. \& Takashi, M. (2004). Autonomic Nervous System Activities During Motor Imagery in Elite Athletes. Journal of Clinical Neurophysiology, 21(3), 170-179. DOI: https://doi.org/10.1097/00004691-200405000-00005

Kazuo, O. Kasai, T. \& Maeshima, T. (2000). Aotunomic Response Specificity during Motor Imagery. Journal of Physiological Anthropology and Applied Human Science, 19(6), 255-261. DoI: https://doi.org/10.2114/jpa.19.255

Lauer, M. (2009, April). Autonomic function and prognosis. Cleveland Clinic Journal of Medicine, 76(2), S18-S22. Dor: 10.3949/ccjm.76.s2.04

Masshour, G. \& Engelhard, K. (2019). Neuroscience and Anaesthesiology. Editorial Oxford.

Pinto, T., Russo, M., Lemos, T., Domingues, C. \& Aureliano, L. (2017). Is heart rate variability affected by distinct motor imagery strategies? Physiology \& Behavior, 189-195. DoI: https://doi.org/10.1016/j.physbeh.2017.05.004

Schmitt, L., Regnard, J. \& Millet, G. (2015). Monitoring Fatigue Status with HRV Measuring in Elite Athletes: An Avenue Beyond RMSSD. Frontiers in Physiology, 6(343). DoI: 10.3389/fphys.2015.00343

Scott, M., Graham, K. \& Davis, G. (2017, May 29). Cardiac Autonomic Responses during Exercise and Post-exercise Recovery Using Heart Rate Variability and Systolic Time Intervals - A Review. Frontiers in Physiology. DoI: https://doi. org/10.3389/fphys.2017.00301 
Tarvainen, M., Lipponen, J., Niskanen, J. \& Ranta-aho, P. (2019, January 14). Kubios HRV User's Guide. Available at www.kubios.com / support@kubios. com DOI: https://doi.org/10.4324/9781315372921-7 